\title{
Enhancement and reproducibility of high quality factor, one-dimensional photonic crystal/photonic wire (1D PhC/PhW) microcavities
}

\author{
N. Nawi ${ }^{1}$, B. Y. Majlis ${ }^{1}$, M. A. Mahdi ${ }^{2}$, R. M. De La Rue ${ }^{3}$, M. Lonçar ${ }^{4}$ and A. R. Md Zain ${ }^{1,4^{*}}$
}

\begin{abstract}
Background: The production of compact and multi-functional photonic devices has become a topic of major research activity in recent years. Devices have emerged that can be used for functional requirements in high speed optical data processing, filtering, nonlinear optical functions such as all-optical switching - and many other applications. The combination of photonic crystal (PhC) structures consisting of a single row of holes embedded in a narrow photonic wire (PhW) waveguide realised in high index-contrast materials is a possible contender for provision of a range of compact devices on a single chip. This trend has been motivated by the availability of a silicon technology that can support monolithic integration to form fully functional devices on CMOS chips.
\end{abstract}

Results: We have successfully demonstrated experimentally an enhancement of the quality factor of a one-dimensional (1D) photonic crystal/photonic wire (PhC/PhW) microcavity that can exhibit resonance quality factor (Q-factor) values as high as 800,000 - together with a low modal volume of approximately $0.5(\mathrm{~N} / \mathrm{n})^{3}$. These results are based on the use of a mode matching approach previously used for device design - through the engineering of tapered hole sections within and outside the cavity - and were achieved without removing the silica cladding layer below the silicon waveguide core. The simulation results obtained in this case also agree with the experimental results obtained.

Conclusions: In this work we have demonstrated that the mode matching, as light enters the photonic crystal structure, can be further enhanced through the use of careful fine tuning of the third hole, $t_{3}$ of the tapered hole region outside the cavity. The Q-factor value obtained was approximately four times greater than that achieved in our previous work on a similar structure.

Keywords: Photonic crystal, Nanophotonic, Integrated optics

\section{Background}

The high index contrast between the silicon and its surrounding cladding makes it possible to design more complex structures - such as sharp bends, abrupt Y-junctions, Mach-Zehnder structures [1-3] - and sensors [4-6]. On the other hand, the large thermo-optic coefficient of silicon also makes it possible to manipulate the refractive index by

\footnotetext{
*Correspondence: rifai@ukm.edu.my

${ }^{1}$ Institute of Microengineering and Nanoelectronics (IMEN), University Kebangsaan, (UKM), 43600 Bangi, Selangor, Malaysia

${ }^{4}$ Harvard John A Paulson, School of Engineering and Applied Science,

Harvard University, Cambridge, MA 02138, USA

Full list of author information is available at the end of the article
}

means of both thermo-optical and electro-optical effects in order to create compact modulators that have been demonstrated in both $\mathrm{PhC}$ and $\mathrm{PhW}$ formats $[7,8]$. The race towards producing microcavity device structures that exhibit high Q-factors and large Purcell-factors has accelerated in recent years. A key aspect is the capabilities of the $\mathrm{PhC} / \mathrm{PhW}$ device structure - which can exhibit a high resonance quality factor (Q-factor) value and strong optical confinement in a small volume, V, for optical signal processing and filtering purposes - implying a large Purcell factor [9-11]. The Q-factor of a resonator is defined as the ratio of the total energy stored in the cavity to the energy 
loss per cycle [12], where $Q=\omega_{0} / \Delta \omega$ [ratio of energy stored to energy loss per cycle]. It can be re-expressed as: $Q=\lambda_{0} /$ $\Delta \lambda$, where $\lambda_{0}$ is the central wavelength of the resonance and $\Delta \lambda$ is the full-width half-maximum (FWHM) value of the resonance, as reported in detail in references [13] and $[14,15,16]$ - where $Q=\lambda_{0} / \Delta \lambda$ implies that the energy stored in the cavity decays exponentially in time, with a decay-time, $\mathrm{t}=\mathrm{Q} / \omega_{0}$. In other words, the quality factor, $\mathrm{Q}$, gives a direct measure of the cavity resonance lifetime. Moreover, the Q-factor values obtained are in accord with the mirror reflectivity, as indicated in [17]. The light confinement can also be characterised using a classical Fabry-Perot model, in which a guided Bloch mode in a $\mathrm{PhC}$ waveguide bounces between the two associated mirrors that form the micro cavity $[18,19]$ - where the reflectivity for the Bloch mode is very close to unity, with estimated reflectivity, $\mathrm{R}, \sim 0.999$.

In photonic crystal $(\mathrm{PhC})$ microcavity structures, the optical properties may usefully be characterized by the $\mathrm{Q} / \mathrm{V}$ ratio (often called the Purcell factor [20, 21]), where $Q$ is the resonance quality factor and $\mathrm{V}$ is the modal volume corresponding to the particular microcavity and its characteristic resonant electromagnetic modes. Designing high Qfactor optical microcavities where the light is confined in a small volume, $\mathrm{V}$, is potentially useful for high speed optical processing - with light being confined within a small volume, on the order of $(\lambda / 2 n)^{3}$ - where $\lambda$ is the emission wavelength and $n$ is the refractive index of the given material. In the present work, the microcavity structures were realized in silicon-on-insulator (SOI). Such silicon based structures are potentially useful in nonlinear processing where the wavelength of the light used is sufficiently long for transparency. By embedding suitable emission capabilities into the high-index medium, the large Purcell factor values that can be achieved, in combination with embedded emission capabilities, e.g. emission obtained by using photo-pumped quantum dots, will enable very efficient and spectrally narrow photon generation.

A variety of different design configurations for achieving high quality factor values have been described in the literature - such as micro-pillars [22-24], silica toroids [25], $\mathrm{PhC}$ cavities [26-31] in photonic wire waveguides - and cavities based on 2D PhC membrane structures [32, 33]. Q-factor values as large as $10^{8}$ have been obtained in silica toroids [34], but their structure has a relatively large modal volume. The interest in manufacturing ultra small microcavity device structures has been boosted by the possibility of achieving modal volumes that are comparable with the notional limiting value of $\mathrm{V}=(\lambda / 2 \mathrm{n})^{3}$ [35]. Based on this motivation, high Q-factor values, greater than $10^{6}$, have been achieved [36, 37, 38] - as well as in other configurations such as 1D PhC micro-cavities [28-31, 39], L3 cavities within 2D PhC environments - where three holes in a line are removed to provide a micro cavity [40] - and also heterostructure microcavities [41, 42]. Our design has demonstrated the large enhancement in the Q-factor that is achievable for light confined within a small volume through the use of the mode matching mechanism where the light with mode profile matching propagates smoothly from the unpatterned waveguide into the periodic section, as discussed in detail in $[18,19]$. But, in most cases, the footprint that the design covers is much larger, especially for 2D PhC based cavities. Therefore, in order to design compact devices that require small footprints while satisfying the requirement for integration with other optical devices, the $1 \mathrm{D} \mathrm{PhC/PhW}$ device was proposed by Foresi et al. in [30]. The footprint of this particular device covered an area of approximately $1 \mu \mathrm{m} \times 3 \mu \mathrm{m}$. Another important factor that needs to be considered in such devices is the reproducibility of the fabrication process that is required for production of device structures with a consistent combination of Q-factor value and cavity resonance frequency [43-45]. In this paper we report on ultra high quality factor 1D photonic crystal/photonic wire $\mathrm{PhC} / \mathrm{PhW}$ device structures that exhibit the high mirror reflectance value of approximately 0.999 , as calculated in [19]. These results were achieved experimentally over different fabrication cycles and samples, through a range of time scales. In this case, we have successfully demonstrated that our Q-factor values remain consistent with variation of less than $1 \%$ through different fabrication cycles that were conducted over a 6 months period.

\section{Methods}

\section{Design and FDTD simulations approach}

Planar 1D PhC microcavity structures consisting of two mirrors formed by several holes separated by a spacer have been realized in $500 \mathrm{~nm}$ wide $\mathrm{PhW}$ silicon waveguides. These combined $\mathrm{PhC} / \mathrm{PhW}$ structures were based on SOI material having a silicon thickness of $260 \mathrm{~nm}$ and a $1 \mu \mathrm{m}$ thick $\mathrm{SiO}_{2}$ cladding underneath it, in order to provide sufficient optical isolation [27, 30]. Potentially, complete removal of the silica cladding can enhance the Q-factor value of the microcavity resonance - and so a $1 \mu \mathrm{m}$ silica lower cladding layer can be regarded as being sufficiently thick. An early version of the $1 \mathrm{D} \mathrm{PhC} / \mathrm{PhW}$ microcavity was described by Foresi et al. in 1997 [27], where the design consisted of a photonic crystal $(\mathrm{PhC})$ structure formed by a single row of holes. This $\mathrm{PhC}$ structure was conceptually separated by inserting a gap, thereby creating a microcavity - but the Q-factor value for this device was only 500. Improvements have been made, more recently, that enhance the microcavity Q-factor value by using tapered hole structures of different sizes and spacing. The design considered in the present paper is shown in Fig. 1 and consists of five periodically spaced holes that form Bragg mirrors on each side of the microcavity. 
These holes have a diameter of $350 \mathrm{~nm}$ and a periodic spacing of $370 \mathrm{~nm}$. The mirrors were separated by a short cavity length, $\mathrm{L}_{\mathrm{C}}$ of $400 \mathrm{~nm}$. In order to reduce the modal mismatch between the unpatterned $\mathrm{PhW}$ waveguide and the cavity section - and, within the cavity, the mismatch between the Bragg mirrors and the cavity spacer section, optimized hole tapers based on previous work [30, 31] were used. The structures consist of four aperiodically spaced and tapered hole sections at either end of the cavity, $\mathrm{N}_{\mathrm{TO}}$ and four aperiodically spaced hole taper sections within the cavity, $\mathrm{N}_{\mathrm{TI}}$. The dimensions of the aperiodic holes are given in Fig. 1. Four aperiodically located and tapered holes form the transition sections within the cavity - and have diameters of 170, 180, 166 , and $131 \mathrm{~nm}$ respectively - with center-to-center hole distances of 342, 304, 310, and $290 \mathrm{~nm}$ respectively - whereas the four-hole aperiodic tapered sections outside the cavity have hole diameters of 131, 166, 185 and $170 \mathrm{~nm}$ respectively - with center-tocenter distances of $275,305,314$ and $342 \mathrm{~nm}$ respectively. With proper design, the tapered hole sections at either end of the cavity reduce the overall transmission loss of the microcavity. The patterns were transferred into the silicon substrate using electron-beam lithography (EBL) tool with negative tone hydrogen silsesquioxane (HSQ) resist as a mask. The PhW waveguides were finally etched using Inductively Coupled Plasma (ICP) dry etching to remove unwanted silicon areas - using a combination of $\mathrm{SF}_{6}$ and $\mathrm{C}_{2} \mathrm{~F}_{4}$ gas mixture.

Finite-difference time-domain (FDTD) simulations using commercial software have been carried out, as also reported in $[30,31]$. The reflectivity of the mirrors in this particular device increased significantly as the number of periodically spaced holes in the mirrors increased. Figure $2 \mathrm{a}$ and $\mathrm{b}$ show the variation of $\mathrm{Q}$-factor values and optical transmission through the cavity, ranging over the number of periodic mirrors used - together with aperiodic taper sections outside the cavity, $\mathrm{N}_{\mathrm{TO}}$. For simplicity in our argument, in this particular condition, we have kept the tapering inside the cavity, $\mathrm{N}_{\mathrm{TI}}=4$ and cavity length, $\mathrm{L}_{\mathrm{C}}=400 \mathrm{~nm}$, for both cases. We have only varied the number of holes in the periodic mirror

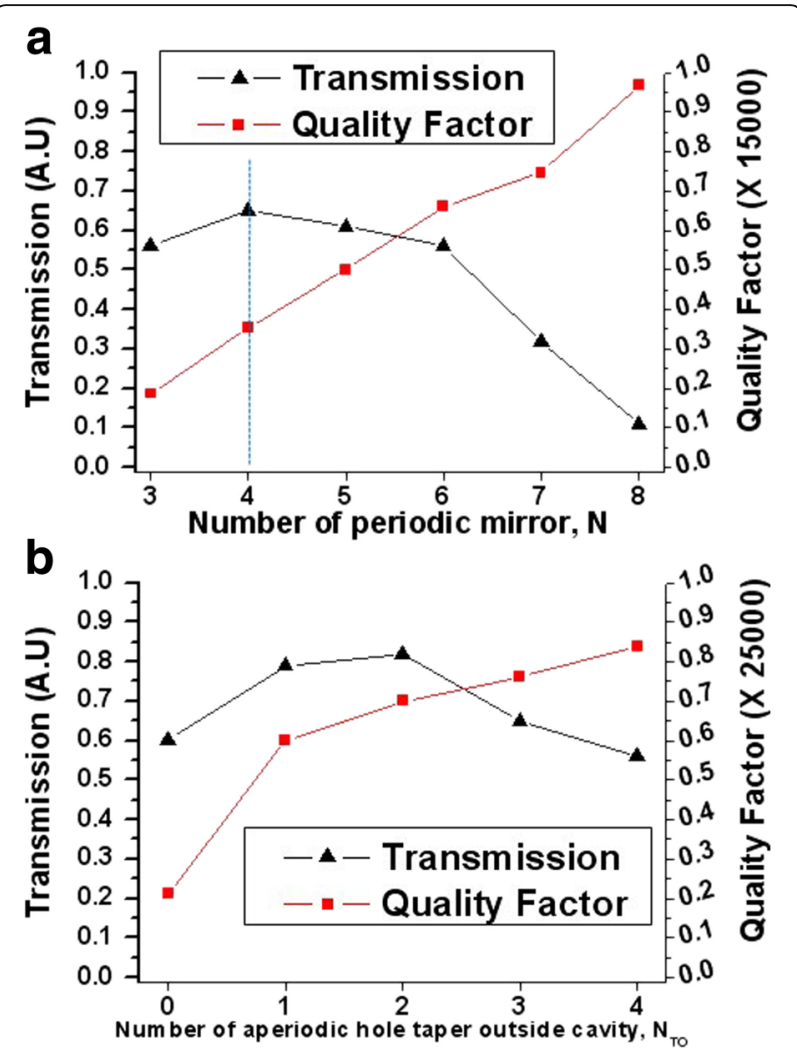

Fig. 2 3D FDTD calculation results for $N_{T I}=4$ and $L_{C}=400 \mathrm{~nm}$ showing the variation of the optical transmission and Q-factor values for: (a) different numbers of periodic mirror holes, without any tapering outside the cavity - and (b) for $N=4$, with different numbers of aperiodic holes in the taper section used outside the cavity, $\mathrm{N}_{\mathrm{TO}}$ in conjunction with the taper design shown in Fig. 1

section, as shown in Fig. 2a and tapering outside cavity, $\mathrm{N}_{\mathrm{TO}}$ as shown in Fig. 2b. For both cases, the Q-factor values show consistent increases as the number of periodically located mirror holes, $\mathrm{N}$, and the number of aperiodically located mirror holes, $\mathrm{N}_{\mathrm{TO}}$, increases. This increase in the Q-factor for an increase in the number of holes in the aperiodic taper sections within the cavity, at a fixed number of periodically located holes, may be attributed to the reduction of the modal mismatch between the unstructured photonic-wire waveguides and the mirror regions. The aperiodic taper sections on each side of the Bragg mirrors outside the cavity on the other

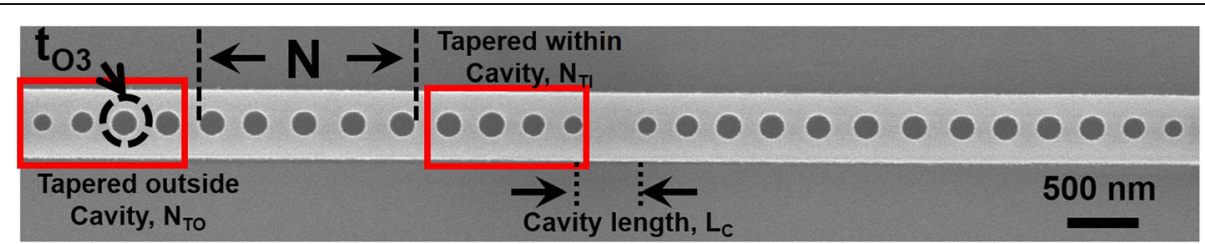

Fig. 1 Scanning electron micrograph (SEM) images of the Silicon on Insulator (SOI) based PhW waveguide with tapered PhC hole structure for a simple microcavity embedded in it with $N=5$, cavity length, $L_{c}=400 \mathrm{~nm}$, Number of tapered holes within the cavity, $N_{T 1}=4$ and number of tapered hole outsides cavity, $\mathrm{N}_{\mathrm{TO}}=4$ 
hand would be expected to reduce the transmission loss through the whole cavity. In all cases, the optical transmission reported in this paper is normalized to the input power of the simulated structures.

\section{Results and Discussions}

\section{Optimizing the Q-factor of microcavities using hole size} and position variation

Further optimization of the structures was carried out on the basis of the design shown in Fig. 1. We have simulated the device structures and compared them on the basis of the number of periodic holes in the middle section, $\mathrm{N}$, together with the number of tapered holes in the section outside the cavity, $\mathrm{N}_{\mathrm{TO}}$ - in order to enhance the optical transmission - as mentioned in our previous work [30]. For simplicity, we have kept constant the parameters used in specifying the tapering within the cavity, $\mathrm{N}_{\mathrm{TI}}$. Based on Fig. 3a, we have demonstrated that in the cases where the number of tapered section holes outside the cavity, $\mathrm{N}_{\mathrm{TO}}$, is 3 and 4 respectively, that - as the number of periodic holes, $\mathrm{N}$, increases - the optical transmission is reduced significantly, probably because of the greater sidewall roughness and discontinuity scattering losses associated with the larger number of holes introduced into the mirror structure. In each case, by varying the radius of the third hole, $t_{3}$, counting from where the light enters into the tapered and aperiodic sections of the PhC microcavity - in the tapered hole section outside the cavity, i.e. the $\mathrm{N}_{\mathrm{TO}}$ section - we have obtained close control of the optical transmission and Q-factor values of the device structure. As shown in the simulation results for the case of $N=7$, together with $\mathrm{N}_{\mathrm{TO}}$ $=4$, we have enhanced the optical transmission by as much as a factor of two, as shown in Fig. 3b - which indicates that the scattering losses are reduced significantly because the light is transmitted smoothly from the un-patterned waveguide into the periodic section. On the other hand, the Q-factor appears to be substantially reduced - which is partly due to the reduction in the optical confinement at the resonance wavelength as light travels through an increased total number of holes within the PhC structures. It is also found that tapering outside the cavity enhances the quality factor via variation of the third hole radius - $t_{3}$ - as shown in Fig. 3b. As the radius of the hole $t_{3}$ was increased from $60 \mathrm{~nm}$ to $105 \mathrm{~nm}$, the optical transmission also increased by $40 \%$. But the Q-factor value was reduced from 540,000 to approximately 320,000 for the case of $t_{3}$ values of $60 \mathrm{~nm}$ and $105 \mathrm{~nm}$ respectively. It can be deduced that the third hole, $t_{3}$, has a small but significant impact via reduction of the propagation losses that occur as the light enters into the periodic mirror regions, thereby enhancing the optical transmission as well as Q-factor values. Based on the results of the FDTD simulations shown in Fig. 3b, the increase in the radius of $t_{3}$ from $60 \mathrm{~nm}$ to $105 \mathrm{~nm}$ has improved the optical transmission by $50 \%$, but there is an accompanying reduction in the Q-factor by 220,000 - from 540,000 to 320,000 .

This behaviour is also shown in the mode profile distribution at the resonance wavelength, for the case of $\mathrm{N}_{\mathrm{TO}}=4$, in Fig. 4a where $N=4$ and Fig. $4 \mathrm{~b}$ for $N=7$ - where the intensity of the optical transmission decreases as the number of periodic holes in the $\mathrm{PhC}$ mirror structures increases from $\mathrm{N}=4$ to $N=7$. The intensity of the mode distribution within the cavity at resonance is shown by the scales on the right and is indicated by different colours, together with absolute values. These results lead to the deduction that the optical transmission is substantially higher for the case of $\mathrm{N}=4$ than for the case of $\mathrm{N}=7$ - which is partly due to the light undergoing greater scattering losses as it travels through an increased number of periodic holes - as shown in Fig. 4c. In contrast, for the case of a radius of $t_{3}=95 \mathrm{~nm}$, the Q-factor increases from 150,000 to approximately 740,000 , as the number of periodic holes increases from $\mathrm{N}=4$ to $N=6$. But the Q-factor was reduced to approximately 300,000 when the number of periodic mirror holes was increased to $\mathrm{N}=7$. We have also shown in the previous section in Fig. 3b that, for the case of $\mathrm{N}$ $=7$, the $\mathrm{Q}$-factor value increased from approximately 300,000 to 540,000 when the radius of $t_{3}$ was reduced to $65 \mathrm{~nm}$.

This trend is partly due to the increase in the optical confinement within the cavity as the number of holes in each Bragg mirror section increases - meaning that the
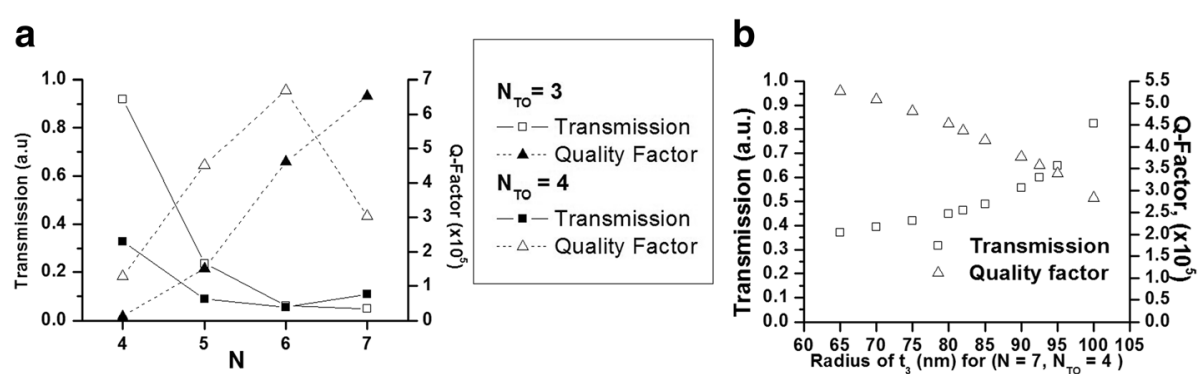

Fig. 3 The simulation results for optical transmission and Q-factor values, obtained using a 3D FDTD approach, for: (a) different numbers of periodic mirror holes, $\mathrm{N}$ - and (b) different radii of the third hole, $\mathrm{t}_{3}$, in the tapered hole section outside the cavity, $\mathrm{N}_{\text {TO }}$ 

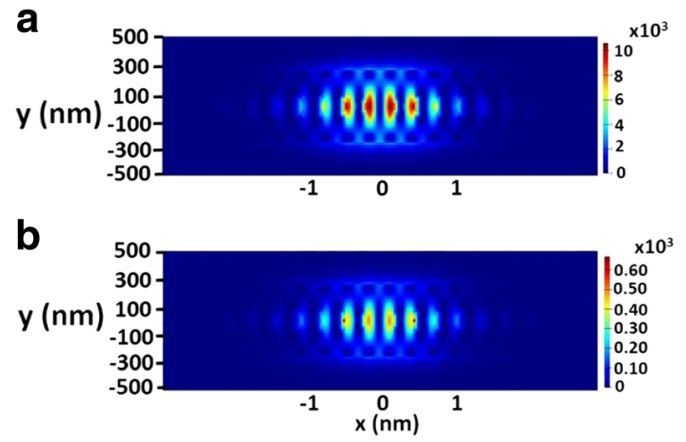

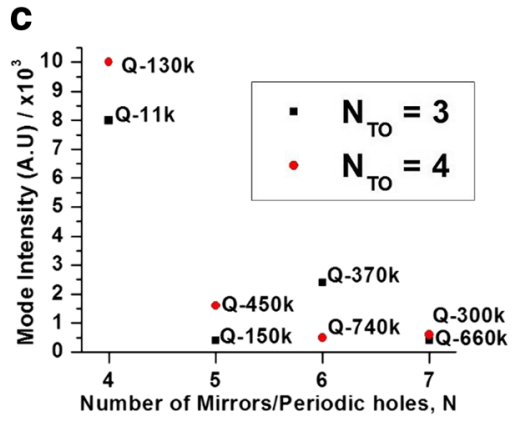

Fig. 4 The mode profile at the resonance wavelength for the case of: a $t_{3}$ diameter of $185 \mathrm{~nm}$ for $(\mathbf{a}) N_{T O}=4, N=4,(\mathbf{b}) N_{T O}=4, N=7$ and mode intensity for $\mathrm{N}$ number of holes with (c) different number of tapered outside cavity, $\mathrm{N}_{\text {TO }}=3$ and $\mathrm{N}_{\text {TO }}=4$

optical energy at the resonance wavelength is trapped longer within the cavity. In addition, It is also found that the optical transmission is always low for the case of $\mathrm{N}_{\mathrm{TO}}=4$, as compared with the case of $\mathrm{N}_{\mathrm{TO}}=3$ - the scattering losses at the interface between the third and fourth holes within the tapered hole section outside the cavity, $\mathrm{N}_{\mathrm{TO}}$, increase as light travels from one hole to another, before entering the periodic mirror section, with $\mathrm{N}$ being the number of holes in the periodic mirror section.

Increasing the number of tapered holes outside the cavity, $\mathrm{N}_{\mathrm{TO}}$, leads to more pronounced secondary resonances appearing at a wavelength of around approximately $1340 \mathrm{~nm}$ - as shown in the inset in Fig. 5. We can deduce from this result that the central resonance with a very high Q-factor value was obtained as the band-gap decreased and clear secondary resonances were observed - essentially forming the band-edge in the shorter wavelength region.
In addition, after optimizing the device structures, we have obtained high Q-factor values, but with reduced optical transmission - in agreesment with our previous analysis, where we obtained a reasonably high optical transmission level, together with high Q-factor values. These Q-factor values were obtained with $N=6$ and with $\mathrm{N}_{\mathrm{TO}}$ and $\mathrm{N}_{\mathrm{TI}}$ having 4-hole sections, as used previously, together with small variations in the value of $t_{3}$, as shown in Fig. 5. The Q-factor obtained using the 3D FDTD approach has been calculated as approximately 740,000 (740 K), together with a normalized simulated transmission of around 30\%. Increasing $\mathrm{N}_{\mathrm{TO}}$ has influenced the Q-factor because the light propagated more smoothly into the periodic Bragg-mirror sections - although the scattering losses have reduced the total optical transmission of the microcavity.

Based on our simulation results, shown in Fig. 5 for the case of $\mathrm{N}=6$ and $\mathrm{N}_{\mathrm{TO}}=\mathrm{N}_{\mathrm{TI}}=4$, where we
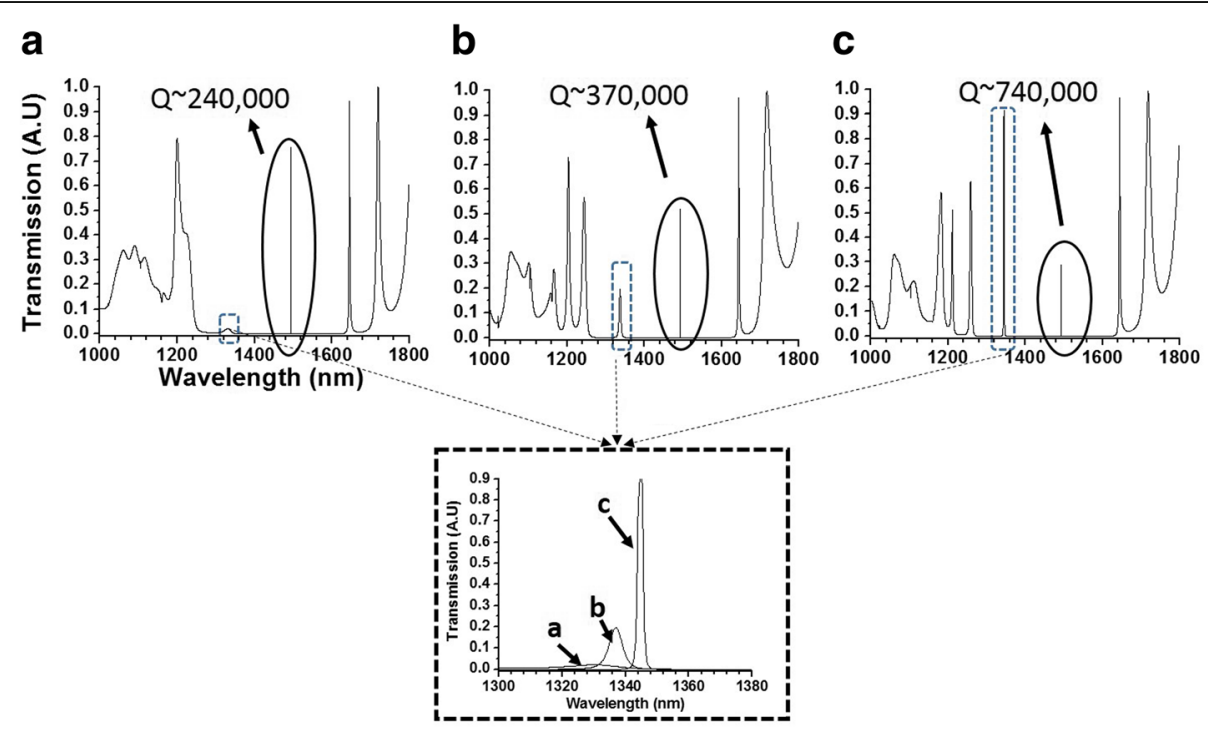

Fig. 5 Simulation results obtained using a 3D FDTD approach for analysis corresponding to the measured results obtained in Fig. 7- where high Q-factor values were obtained for $N=6$, with $\mathrm{N}_{T 1}=4$ and (a) $\mathrm{N}_{\text {TO }}=0$, FWHM $\sim 6 \mathrm{pm}$, (b) $\mathrm{N}_{\text {TO }}=3$, FWHM $\sim 4 \mathrm{pm}$ (c) $\mathrm{N}_{\text {TO }}=4$, FWHM 2 pm, at a resonance wavelength of approximately $1498.338 \mathrm{~nm}$. Inset is the second resonance excited at the wavelength of approximately $1340 \mathrm{~nm}$ 
have varied the specified diameter of the third hole, $t_{3}$, (as illustrated in Fig. 1) - we have enhanced the Q-factor to a value of approximately 800,000 at a resonance frequency of $1498.465 \mathrm{~nm}$, with a FWHM of approximately $1.8 \mathrm{pm}$, as shown in Fig. 6c. Figure 6 also shows the variation of the $\mathrm{Q}$-factor value for three different arrangements of the tapered holes outside the cavity, $\mathrm{N}_{\mathrm{TO}}$, while keeping the diameter of $t_{3}$ at $185 \mathrm{~nm}$. Figure $6 \mathrm{a}$ and $\mathrm{b}$ show Q-factor values of approximately 115,000 and 150,000 obtained for the case of $\mathrm{N}_{\mathrm{TO}}=0$ and $\mathrm{N}_{\mathrm{TO}}=3$, respectively. Furthermore, the reductions in the Q-factor value in Fig. 6 are partly due to the modal mismatch between the unpatterned wire and the $\mathrm{PhC}$ region. The measured results were obtained using a tunable laser covering the range from $1480 \mathrm{~nm}$ to $1600 \mathrm{~nm}$ - and the optical transmission values obtained were normalised against the transmission of and unpatterned wire without any mirrors embedded in it.

Figure 7 shows a more detailed comparison between the simulated and measured results discussed previously. The High Q-factor value of approximately 800,000 was measured, as indicated in Fig. $7 \mathrm{~b}$, for the case of $N=6$, $\mathrm{N}_{\mathrm{TO}}=4$, with hole $\mathrm{t}_{3}$ having an optimized diameter of $185 \mathrm{~nm}$, i.e. a radius of $92.5 \mathrm{~nm}$. The optical transmission is normalised against the unpatterned wire waveguide without any hole embedded in it. In addition, the Q-factor was calculated using the FDTD approach - with the value of approximately 740,000 , as shown in Fig. 7 a. The resonance was shifted by approximately $25 \mathrm{~nm}$ for the measured device, as compared with the simulation result - which is partly due to variations in the actual dimensions obtained after carrying out the fabrication processes. Apart from that, during the simulation, only the horizontal hole tapering is considered - whereas, in the fabrication process, tapering may also occur vertically since the etching rate in each hole of the taper is different because the etching process is slower in small holes, as compared with bigger holes, and possibly results in tapering in the vertical direction, inside the hole.

A proper assessment of the performance of microcavities depends on two crucial parameters - which are the resonance quality factor, $Q$, and the modal volume, $V$ [13] - where the Q-factor value directly determines the photon lifetime within the cavity and the modal volume, $V$, is the key factor for assessing the impact of the electromagnetic confinement of the mode within the cavity. The Q-factor value obtained in a small volume, $V$, also implies a large value for the Purcell factor, $F_{p}$, which is given by the dimensionless general expression based on $[20,21]$;

$$
F p=\frac{3}{4 \pi^{2}}\left(\frac{\lambda}{n}\right)^{3}\left(\frac{Q}{V}\right)
$$

where $(\lambda / n)^{3}$ is the reference volume defined by the scaled wavelength in the material with refractive index $n$ - and $Q$ and $V$ are the quality factor of the resonance and the resonant modal volume of the microcavity, respectively. In addition, the Purcell factor for an emitter in the resonant cavity can be expressed on the basis of Fermi's golden rule, as discussed in $[46,47]$ - thereby giving the Purcell factor as the enhancement of the spontaneous emission rate for the microcavity with respect to the spontaneous emission rate into free space as;

$$
F p=\frac{\Gamma}{\Gamma_{0}}=\frac{6 Q(\lambda / 2 n)^{3}}{\pi^{2} V_{e f f}}=\frac{6 Q}{\pi^{2} \tilde{V} e f f}
$$

Where $n$ is the refractive index at the resonance frequency, $V_{\text {eff }}$ is the effective volume of the electromagnetic mode within the cavity and $\tilde{V}_{\text {eff }}$ is the normalized dimension-less effective index volume [48]. This is true

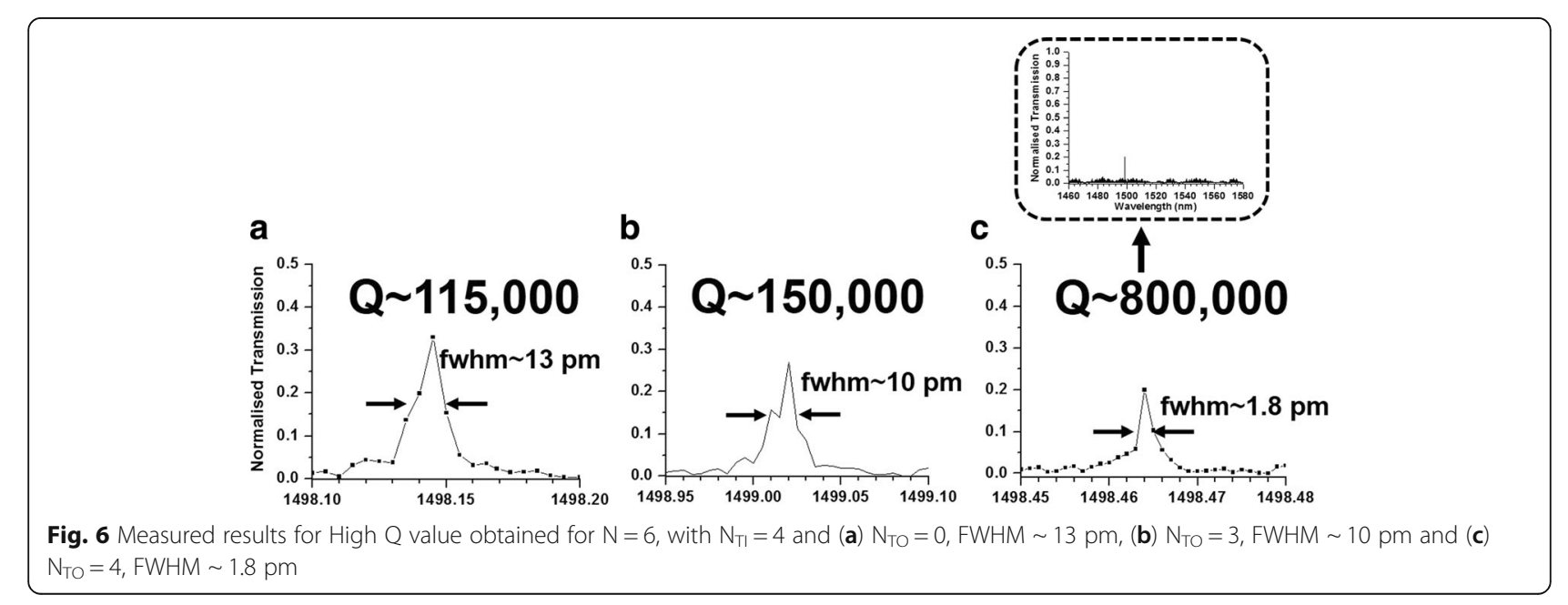



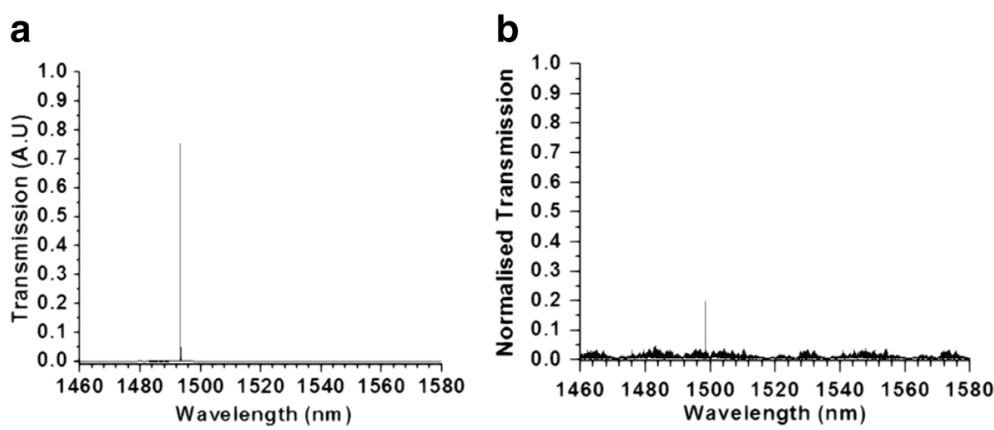

Fig. 7 A detailed comparison between the highest Q obtained using (a) FDTD simulations approach, and (b) Measured results- normalized against the un patterned wire waveguide without any holes embedded in it-, for the case of $\mathrm{N}=6, \mathrm{~N}_{\mathrm{TO}}=4$ and the optimized $\mathrm{t}_{3}$ with diameters of approximately $185 \mathrm{~nm}$

for $V_{\text {eff }}$ when the condition is such that the linewidth of the cavity resonance is greater than the emission linewidth - where, in this case, the $F_{p}$ value is strongly affected by the value of the Q-factor. But in the case where the resonance linewidth is smaller than the emission linewidth, the Q-factor value will be highly influenced by the reduction in the normalized effective volume, $\tilde{V}_{\text {eff }}$. The normalized mode volume $\tilde{V}_{\text {eff }}$ can therefore be defined as:

$$
\tilde{V}_{\text {eff }}=V_{\text {eff }}\left(\frac{2 n}{\lambda}\right)^{3}
$$

Therefore, on the basis of Eq. 3, the Purcell factor of the microcavity can be estimated. In this work, we have embedded the custom $\mathrm{PhC}$ holes within the waveguide cross-sectional area of $500 \mathrm{~nm} \times 260 \mathrm{~nm}^{2}$ - with a refractive index value of 3.48 and a lower cladding refractive index of approximately 1.46 [27]. The finitedifference time-domain (FDTD) approach was used to calculate the Q-factor of the resonant mode, agreeing with the measured value of $\mathrm{Q}$, and is approximately 800,000 . Based on Eq. 3, the value of the normalized modal volume estimated from simulation for this particular device was approximately $0.5(\lambda / n)^{3}$. We estimate that the value of the $\mathrm{Q} / \mathrm{V}$ ratio in this case is approximately $1.6 \times 10^{6}(\lambda / \mathrm{n})^{-3}$. In comparison, Song et al. [36] have obtained a resonance $\mathrm{Q}$-factor value of approximately 600,000 , together with a modal volume of approximately $1.2(\lambda / \mathrm{n})^{3}$ and a resulting $\mathrm{Q} / \mathrm{V}$ value of approximately $5 \times 10^{5}(\lambda / \mathrm{n})^{-3}$. Velha et al. have achieved a resonant modal volume of approximately 0.6 $(\lambda / \mathrm{n})^{3}$ with 1D PhC structures, together with a Q-factor value of approximately 8900 [28] - and a resulting $\mathrm{Q} / \mathrm{V}$ value of approximately $1.5 \times 10^{4}(\lambda / \mathrm{n})^{-3}$. We believe that a higher Q-factor value for the case of the 1D PhC microcavity based on Velha et al. could be obtained, together with modal volume values close to the theoretical limit of $V=(\lambda / 2 n)^{3}$, by tuning the tapered hole structures both outside and within the cavity. Reducing the effective modal volume will also increase the spontaneous emission rate $[18,19]$ of a device that is capable of light emission. Furthermore, we believe that even higher $\mathrm{Q} / \mathrm{V}$ and normalized Purcell factor values can be realized by using the slot-guide approach that is described in Robinson et al. [48].

\section{Conclusions}

Our demonstration of obtaining high resonance Q-factor values in $1 \mathrm{D} \mathrm{PhC} / \mathrm{PhW}$ microcavity device structures has also shown that the fabrication processes for such device structures can be usefully reproducible - as demonstrated for a number of different samples and different process runs over an extended period of time. The effect of the Fabry-Perot (FP) fringes contributed by the cleaved endfacets appears to be suppressed when accompanied by the highest Q-factor values produced by the microcavity itself. This is observed in our experimental results with a high Q-factor value of approximately 800,000 at a resonance wavelength of $1498.46 \mathrm{~nm}$, as shown in Fig. $6 \mathrm{c}$ and when compared with our previous reported results - although the overall FP effects due to the end facets can still be clearly seen throughout the whole spectrum. We have successfully shown that, through several different fabrication cycles using different samples, it is possible to produce consistent values of resonance Q-factor and resonance frequency.

Different measurement cycles for each of the samples have also shown that consistent values for the Q-factor and resonance frequency were preserved. At this point, the effect of intra-chip variation on the $\mathrm{Q}$-factor values produced has not yet been fully assessed. Our studies have focused primarily on reproducing the high Q-factor values for different cycles with one specific design - and completely different samples and designs will be required to determine the impact of environmental effects such as air pressure and humidity variations. The highest measured Q-factor value obtained for the $1 \mathrm{D} \mathrm{PhC} / \mathrm{PhW}$ 
microcavity device was 800,000 - and this was obtained via small variations in the tapered hole structures outside the cavity. We have also demonstrated that the third hole, $t_{3}$, within the tapered section outside the microcavity exhibits a desirably significant impact on the optical transmission and quality factor values - where a $35 \mathrm{~nm}$ difference in that particular hole radius gave an approximately factor of two increase in optical transmission but also a substantially reduced Q-factor, going from 550,000 down to approximately 340,000 .

For instance, the measured results show good agreement with the simulated results obtained by using the FDTD approach. In conclusion, we have successfully designed and fabricated ultra high Q-factor microcavity structures, with a Q-factor value of approximately 800,000 , at the very low modal volume of approximately $0.5(\lambda / \mathrm{n})^{3}$ and an actual volume calculated of approximately $0.015 \mu \mathrm{m}^{3}$. These values gave an estimated $\mathrm{Q} / \mathrm{V}$ ratio of approximately $1.6 \times 10^{6}(\lambda / \mathrm{n})^{-3}$ - which, to our knowledge, is the highest measured $\mathrm{Q} / \mathrm{V}$ ratio for a structure based on an SOI substrate. It is the combination of a very high $\mathrm{Q}$-factor value and a very small modal volume that is outstanding for this particular microcavity geometry. While higher Q-factor values have been obtained elsewhere, the modal volume in our structure is invariably, to the best of our knowledge, the smallest demonstrated in a $\mathrm{PhC}$ based resonator structure.

\section{Abbreviations}

PhC: Photonic crystal; PhW: Photonic wire

\section{Acknowledgments}

This work was supported in part by the Ministry of Higher Education Malaysia (MoHE) through LRGS (2015)/NGOD/UM/KPT and LRGS/2015/UKMUKM/NANOMITE/04/01.

\section{Funding}

Ministry of Higher Education Malaysia (MoHE) through LRGS (2015)/NGOD/ UM/KPT and LRGS/2015/UKM-UKM/NANOMITE/04/01

\section{Availability of data and materials}

Not Applicable.

\section{Authors' contributions}

All Authors read and approved the final manuscript.

\section{Competing interests}

All authors declare that they have no competing interests.

\section{Publisher's Note}

Springer Nature remains neutral with regard to jurisdictional claims in published maps and institutional affiliations.

\footnotetext{
Author details

${ }^{1}$ Institute of Microengineering and Nanoelectronics (IMEN), University Kebangsaan, (UKM), 43600 Bangi, Selangor, Malaysia. ${ }^{2}$ Wireless and Photonic Network Research Center, Faculty of Engineering, University Putra UPM, 43400 Serdang, Selangor, Malaysia. ${ }^{3}$ School of Engineering, Rankine Building, Oakfield Avenue, University of Glasgow, Glasgow G12 8LT, UK. ${ }^{4}$ Harvard John A Paulson, School of Engineering and Applied Science, Harvard University, Cambridge, MA 02138, USA.
}

Received: 11 September 2017 Accepted: 29 January 2018

Published online: 20 February 2018

\section{References}

1. Ahmad, R.U., Pizzuto, F., Camarda, G.S., Espinola, R.L., Rao, H., Osgood, R.M. Ultra compact corner-mirrors and T branches in silicon-on- insulator. IEEE Photon. Technol. Lett. 14(1), 65-67 (2002)

2. Ohno, F., Fukuzawa, T., Baba, T.: Mach-Zehnder interferometers composed of $\mu$-bends and $\mu$-branches in a Si photonic wire waveguide. Jpn. J. Appl. Phys. 44(7A), 5322-532 (2005)

3. Camargo, E.A., Chong, H.M.H., De La Rue, R.M.: Highly compact asymmetric mach-Zehnder device based on channel guides in a two-dimensional photonic crystal. Appl. Opt. 45, 6507-6510 (2006)

4. Yang, D., Tian, H., Ji, Y., Quan, Q.: Design of simultaneous high-Q and highsensitivity refractive index sensor. J. Opt. Soc. Am. B. 30(8), 2027-2031 (2013)

5. Yang, D., Kita, S., Liang, F., Wang, C., Tian, H., Ji, Y., Loncar, M., Qimin, Q.: High sensitivity and high Q-factor nanoslotted parallel quadrabeam photonic crystal cavity for real-time and label-free sensing. Appl. Phys. Lett. 105(6), 063118 (2014)

6. Chen, Y.F., Xavier, S., Rupa, S., Peng, C., Erickson, D.: Controlled photonic manipulation of proteins and other Nanomaterials. Nano Lett. 12(3), 16331637 (2012)

7. Chong, H.M.H., De La Rue, R.M.: Tuning of photonic crystal waveguide microcavity by thermooptic effect. IEEE Photon. Technol. Lett. 16(6), 15281530 (2004)

8. Geis, M.W., Spector, S.J., Williamson, R.C., Lyszczarz, T.M.: Submicrosecond submilliwatt silicon-on-insulator thermooptic switch. IEEE Photon. Technol. Lett. 16(11), 2514-2516 (2003)

9. Belotti, M., Galli, M., Gerace, D., Andreani, L.C., Guizzetti, G., Md Zain, A.R., Johnson, N.P., Sorel, M., De La Rue, R.M.: All-optical switching in silicon-oninsulator photonic wire nano-cavities. Opt. Express. 18(2), 1450-1461 (2010)

10. Yeh, P.: Optical Waves in Layered Media. J. WILEY EDITIONS, ISBN: 978-0-47173192-4 (1988)

11. Haus, H.A.: Waves and Fields in Optoelectronics. Prentice Hall, ISBN-13: 9780139460531 (1984)

12. Jackson, J.D.: Classical Electrodynamics, 2nd edn. John Wiley, New York (1974)

13. Lalanne, P., Sauvan, C., Hugonin, J.P.: Photon confinement in photonic crystal nano-cavities. Laser Photo. Rev. 1-13 (2008) Wiley Inter-Science

14. Bogaerts, W., De Heyn, P., Vaerenbergh, T.V., De Vos, K., Selvaraja, S.K., Claes, T., Dumon, P., Bienstman, P., Van Thourhout, D., Baets, R.: Silicon ring resonators. Laser. Photo. Rev. 6(1), 47-73 (2012)

15. Md Zain, A.R., Johnson, N.P., Sorel, M., De La Rue, R.M.: Design and fabrication of high quality factor 1-D photonic crystal/photonic wire extended microcavities. IEEE Photon. Technol. Lett. 22, 9 (2010)

16. Husna, J., Mohamed, M.A., Sampe, J., Md Zain, A.R.: Numerical simulation of one dimensional (1D) photonic crystal multiple cavities based on silicon on insulator (SOI). Micro and Nanoelectronics (RSM), 2015 IEEE Regional Symposium on Semiconductor, 1-4, (2015)

17. Lermer, M., Gregersen, N., Dunzer, F., Reitzenstein, S., Höfling, S., Mørk, J., Worschech, L., Kamp, M., Forchel, A.: Bloch wave engineering of quantum dot micropillars for cavity quantum electrodynamics experiments. Phys. Rev. Lett. 108, 057402 (2012)

18. Sauvan, C., Lecamp, G., Lalanne, P., Hugonin, J.P.: Modal-reflectivity enhancement by geometry tuning in photonic crystal microcavities. Opt. Express. 13, 245 (2005)

19. Sauvan, C., Lalanne, P., Hugonin, J.P.: Slow-wave effect and mode-profile matching in photonic crystal microcavities. Phys. Rev. B. 71, 165118 (2005)

20. Purcell, E.M., Torrey, H.C., Pound, R.V.: Resonance absorption by nuclear magnetic moments in a solid. Phys. Rev. 69, 37 (1946)

21. Purcell, E.M.: Spontaneous emission probabilities at radio frequencies. Phys. Rev. 69, 681 (1946)

22. Iga, K., Koyama, F., Kinoshita, S.: Surface emitting semiconductor-lasers. IEEE J. Quantum Electron. 24, 1845-1855 (1988)

23. Reitzenstein, S., Hofmann, C., Gorbunov, A., Gorbunov, M., Strauss, M., Kwon, S.H., Schneider, C., Loffler, A., Hoefling, S., Kamp, M., Forchel, A.: AlAs/GaAs micropillar cavities with quality factors exceeding 150,000. Appl. Phys. Lett. 90, 251109 (2007)

24. Lecamp, G., Hugonin, J.P., Lalanne, P., Braive, R., Varoutsis, S., Laurent, S, Lemaitre, A., Sagnes, I., Patriarche, G., Robert-Philip, I., Abram, I.: Submicrondiameter semiconductor pillar microcavities with very high quality factors. Appl. Phys. Lett. 90, 091120 (2007) 
25. Armani, D.K., Kippenberg, T.J., Spillane, S.M., Vahala, K.J.: Ultralow-threshold microcavity Raman laser on a microelectronic chip. Nature. 421(925), 925928 (2003)

26. Zhang, J.P., Chu, D.Y., Wu, S.L., Bi, W.G., Tiberio, R.C., Joseph, R.M., Taflove, A., Tu, C.W., Ho, S.T.: Nanofabrication of 1-D photonic bandgap structures along a photonic wire. IEEE Photon. Technol. Lett. 8, 491-493 (1996)

27. Foresi, J.S., Villeneuve, P.R., Ferrera, J., Thoen, E.R., Steinmeyer, G., Fan, S., Joannopoulos, J.D., Kimmerling, L.C., Smith, H.I., Ippen, E.P.: Photonic Bandgap microcavities in optical waveguides. Nature. 390, 143-145 (1997)

28. Velha, P., Rodier, J.C., Lalanne, P., Hugonin, J.P., Peyrade, D., Hadji, E.: Ultra high-reflectivity photonic-bandgap mirrors in a ridge SOI waveguide. New J. Phys. 8(204), (2006)

29. Velha, P., Picard, E., Hadji, E., Rodier, J.C., Lalanne, P., Peyrade, D.: Ultrahigh QN Fabry-Perot microcavity on SOl substrate. Opt. Express. 15, 16090-16096 (2007)

30. Md Zain, A.R., Johnson, N.P., Sorel, M., De La Rue, R.M.: Ultra high quality factor one dimensional photonic crystal/photonic wire micro-cavities in silicon-on-insulator (SOI). Opt. Express. 16, 12084-12089 (2008)

31. Md Zain, A.R., De La Rue, R.M.: Control of coupling in 1D photonic crystal coupled cavity nano-wire structures via hole diameter and position variation. J. Opt. 17, 125007 (2015)

32. Akahane, Y., Asano, T., Song, B.S., Noda, S.: High-Q photonic nanocavity in two-dimensional photonic crystal. Nature. 425, 944-947 (2003)

33. Weidner, E., Combrie, S., NVQ, T., De Rossi, A., Nagle, J., Cassette, S., Talneau, A., Benisty, H.: Achievement of ultrahigh quality factors in GaAs photonic crystal membrane nanocavity. Appl. Phys. Lett. 89, 221104 (2006)

34. Armani, D.K., Kippenberg, T.J., Spillane, S.M., Vahala, K.J.: Ultra high-Q toroid microcavity on a chip. Nature. 421, 925-928 (2003)

35. Gondarenko, A., Lipson, M.: Low modal volume dipole-like dielectric slab resonator. Opt. Express. (16), 22 17400-17409 (2008)

36. Song, B.S., Noda, S., Asano, T., Akahane, Y.: Ultrahigh-Q photonic doubleheterostructure nanocavity. Nat. Mater. 4, 207-210 (2005)

37. Deng, C.S., Peng, H.G., Gao, Y.S., Zhong, J.X.: Ultra high Q photonic crystal Nanobeam cavities with H-shaped holes. Physica. E. Low-Dimensional. Syatem. Nanostructures. 63, 8-13 (2014)

38. Liu, H.C., Yariv, A.: Designing coupled resonator optical waveguides basedon high-Q tapered grating-defect resonators. Opt. Express. 20(8), 9249-9263 (2012)

39. Miri, M., Sodagar, M., Mehrani, K., Eftekhar, A.A., Adibi, A., Rashidian, B.: Design and fabrication of photonic crystal Nano-beam resonator: transmission line model. J. Lightwave Technol. 32(1), 91-98 (2014)

40. Kippenberg T. J., Spillane, S. M., Armani, D. K., Vahala, K. J.: Ultralow-threshold microcavity Raman laser on a microelectronic chip. Optics Letters, 11(29), 1224-1226 (2004)

41. Asano, T., Song, B.S., Akahane, Y., Noda, S.: Ultrahigh-Q Nanocavities in TwoDimensional Photonic Crystal Slabs. IEEE Journal of Selected Topics in Quantum Electronics, 6(12), 1123-1134, (2006)

42. Akahane, Y., Asano, T., Song, B.S., Noda, S.: Fine-tuned high-Q photoniccrystal. Opt. Express. 13, 1202-1214 (2005)

43. Shankar, R., Bulu, I., Loncar, M.: Integrated high quality factor silicon-onsapphire ring resonators for the mid-infrared. Appl. Phys. Lett. 102(5), (2013)

44. Lee, H., Chen, T., Li, J., Yang, K.Y., Jeon, S., Painter, O., Vahala, KJ.: Chemically etched ultrahigh-Q wedge-resonator on a silicon chip. Nat. Photonics. 6(6), 369-373 (2012)

45. Gnan, M., Thoms, S., Macintyre, D.S., De La Rue, R.M.: Fabrication of low loss photonic wires in silicon-on-insulator using hydrogen silsesquioxane. Electron. Lett. 44, 115-116 (2008)

46. Confined Electrons and Photons: New Physics and Applications. In: Burstein, E, Weisbuch, C (eds.). Springer US, Plenum, New York (1994)

47. Gayral, B., Gerard, J.M., Lemaitre, A., Dupuis, C., Manin, L., Pelouard, J.L.: HighQ wet-etched GaAs microdisks containing InAs quantum boxes. Appl. Phys. Lett. 75, 1908 (1999)

48. Robinson, J.T., Manolatou, C., Chen, L., Lipson, M.: Ultrasmall mode volumes in dielectric optical microcavities. Phys. Rev. Lett. 95, 143901 (2005)

\section{Submit your manuscript to a SpringerOpen ${ }^{\circ}$ journal and benefit from:}

- Convenient online submission

- Rigorous peer review

- Open access: articles freely available online

- High visibility within the field

- Retaining the copyright to your article

Submit your next manuscript at $\boldsymbol{\nabla}$ springeropen.com 